\title{
Non-pegylated liposomal doxorubicin for patients with recurrent ovarian cancer: A multicentric phase II trial
}

\author{
JANINA BRUCKER $^{1 *}$, CHRISTINE MAYER $^{1 *}$, GERHARD GEBAUER $^{2}$, PETER MALLMANN $^{3}$, \\ ANTJE KRISTINA BELAU ${ }^{4}$, ANDREAS SCHNEEWEISS ${ }^{5}$, CHRISTOF SOHN $^{1}$ and MICHAEL EICHBAUM ${ }^{6}$ \\ ${ }^{1}$ Department of Gynecology and Obstetrics, University of Heidelberg Medical School, D-69120 Heidelberg; \\ ${ }^{2}$ Department of Gynecology and Gynecological Oncology, Marien Hospital Hamburg, D-22087 Hamburg; \\ ${ }^{3}$ Department of Gynecology and Obstetrics, University of Cologne Medical School, D-50931 Köln; \\ ${ }^{4}$ Department of Gynecology and Obstetrics, University of Greifswald Medical School, D-17475 Greifswald; \\ ${ }^{5}$ National Center for Tumor Diseases, University Hospital Heidelberg, D-69120 Heidelberg; \\ ${ }^{6}$ Department of Gynecology, St. Marien Hospital Frankfurt, D-60318 Frankfurt, Germany
}

Received July 12, 2015; Accepted June 2, 2016

DOI: $10.3892 / \mathrm{ol} .2016 .4740$

\begin{abstract}
Patients with non-platinum-sensitive recurrent ovarian cancer have a poor prognosis. Non-pegylated liposomal doxorubicin $\left(\right.$ Myocet $^{\circledR}$ ) is a promising drug that may be able to improve treatment for such patients. In the current study, patients with recurrent ovarian cancer relapsing within 12 months after primary treatment received non-pegylated liposomal doxorubicin at $75 \mathrm{mg} / \mathrm{m}^{2} \mathrm{~d} 1 \mathrm{q} 22$ and $60 \mathrm{mg} / \mathrm{m}^{2} \mathrm{~d} 1 \mathrm{q} 22$ after study dose modification, respectively. There were 29 patients enrolled in the trial, and 124 cycles of non-pegylated liposomal doxorubicin were administered in total. All 29 patients were evaluable for toxicity. The clinical benefit rate (defined as the proportion of patients with either complete remission or partial remission, or with stable disease for $>6$ months) was $50 \%$. The predominant non-hematological toxicity was nausea and vomiting (18 patients, grade I/II), whilst no palmar plantar erythrodysesthesia was observed. In 3 patients, a grade III hematological toxicity occurred, and the treatment schedule
\end{abstract}

Correspondence to: Dr Janina Brucker, Department of Gynecology and Obstetrics, University of Heidelberg Medical School, Im Neuenheimer Feld 440, D-69120 Heidelberg, Germany

E-mail: janina.brucker@med.uni-heidelberg.de

Abbreviations: CA125, cancer antigen 125; CBR, clinical benefit rate; CT, computed tomography; EOC, epithelial ovarian cancer; FIGO, International Federation of Gynecology and Obstetrics; MRI, magnetic resonance imaging; OS, overall survival; PFS, progression-free survival; PPE, palmar plantar erythrodysesthesia; $\mathrm{SD}$, standard deviation

*Contributed equally

Key words: epithelial ovarian cancer, platinum-resistant recurrence, non-pegylated liposomal doxorubicin was consequently modified to $60 \mathrm{mg} / \mathrm{m}^{2} \mathrm{~d} 1 \mathrm{q} 22$. The findings suggest that non-pegylated liposomal doxorubicin administered in a schedule of $60 \mathrm{mg} / \mathrm{m}^{2} \mathrm{~d} 1 \mathrm{q} 22$ is well-manageable and is associated with tolerable non-hematological toxicities (predominantly nausea).

\section{Introduction}

Epithelial ovarian cancer (EOC) is the most lethal type of gynecological malignancy (1). Each year, almost 22,000 new diagnoses of EOC are made in the United States, and $\sim 14,000$ mortalities occur due to disease progression (1). The majority of patients in the early stages of the disease are free of any symptoms; thus, the disease is often of an advanced stage when diagnosed (2). Despite efforts made towards the improvement of primary treatment, over two thirds of treated patients relapse and require a sufficient second-line treatment strategy, which is typically palliative (3).

In cases of platinum-sensitive relapse, the standard treatment approach for tumor recurrence occurring at $\geq 12$ months after the primary platinum-based chemotherapy is a re-challenge of platinum-containing regimens; several large trials have proven superiority of platinum-based chemotherapy as a second line therapy compared to non-platinum base chemotherapy (4-6). By contrast, patients relapsing within the first 6 months after primary treatment are considered to have platinum-resistant disease, and the treatment options, as well as the prognosis of the patients, are limited (7). Patients who experience tumor recurrence between 6 and 12 months after primary treatment are considered to have partially platinum-sensitive disease (8). Thus, the necessity for active and tolerable drugs for patients with recurrent EOC is urgent, particularly for cases of non-platinum-sensitive relapse.

In patients with platinum-refractory EOC, a number of cytostatic agents, including paclitaxel, topotecan and gemcitabine, have been trialled and have demonstrated activity (7-9). Furthermore, pegylated liposomal doxorubicin has been reported to be active and well-tolerable as 
second-line treatment option (10-16). However, pegylated liposomal doxorubicin presents a specific toxicity profile, including palmar plantar erythrodysesthesia (PPE) most prominently, which limits feasibility of this regimen $(17,18)$. Non-pegylated liposomal doxorubicin (Myocet $^{\circledR}$ ) has been studied in clinical trials in the treatment of several types of solid tumor, including metastatic breast cancer (16). Due to its different pharmacological formulation, which lacks a pegylated membrane around the doxorubicin-carrying liposomes, non-pegylated liposomal doxorubicin should cause less PPE. This hypothesis is driven from preclinical experiences (17) and has been confirmed by clinical evidence (12-16). However, few data are currently available on the potential role of this drug in the treatment of recurrent ovarian cancer.

The present prospective multicentric non-randomized single-arm phase II trial was conducted to gain further data regarding the activity and tolerability of non-pegylated liposomal doxorubicin in patients with recurrent ovarian cancer, relapsing within 12 months after primary platinum-containing therapy.

\section{Patients and methods}

Trial design. This prospective non-randomized single-arm phase II trial was a multicenter study in nine German sites. The majority of the patients were treated in the Department of Gynecology and Obstetrics of the University Hospital of Heidelberg (Heidelberg, Germany). The study was designed to assess the toxicity and efficacy of non-pegylated liposomal doxorubicin (Myocet ${ }^{\circledR}$; Cephalon, Inc.; Teva Pharmaceutical Industries, Ltd., Frazer, PA, USA) in patients with recurrent non-platinum-sensitive ovarian cancer, which was defined as relapse within 12 months after the end of platinum-containing first-line chemotherapy. Other studies have shown that patients with recurrent ovarian cancer within 12 months after platinum-based first line chemotherapy do not benefit by a second surgery; these patients should be treated with a second-line chemotherapy (19). The current study was approved by the institutional ethics committee of the University of Heidelberg. All patients signed an informed consent prior to the beginning of treatment.

Treatment plan. All patients received non-pegylated liposomal doxorubicin in a $1-\mathrm{h}$ intravenous (i.v.) infusion every 21 days for a maximum of 6 cycles, except in cases of disease progression during treatment. A prolongation of treatment for a further 2 cycles was allowed following the clinical decision of local investigators. The trial commenced with a dosage of $75 \mathrm{mg} / \mathrm{m}^{2}$. Due to the experience of myelosuppression grade III in 3 of the first 5 treated patients, a dose reduction to $60 \mathrm{mg} / \mathrm{m}^{2}$ d1q22 was implemented and reviewed as an amendment to the trial. Premedication consisted of dexamethasone (8 mg, i.v.) and granisetron (1 mg i.v.). In addition, patients received dexamethasone (4 mg twice daily) on days 2 and 3. Treatment was discontinued in cases of progressive disease or unacceptable toxicity, or as per the patient's preference.

Eligibility criteria. Women aged between 18 and 75 years with histologically confirmed EOC, cancer of the fallopian tube or peritoneal cancer, with a prior therapy consisting of platinum-containing chemotherapy and a second-line chemotherapy situation, were included in this study if the platinum-free interval was $<12$ months. Patients were required to have an evaluable or measurable tumor mass on computed tomography (CT) or magnetic resonance imaging (MRI) scans, or progression of the disease in terms of significant increase of cancer antigen 125 (CA125; >25\% compared to the lowest value prior to the commencement of non-pegylated liposomal doxorubicin therapy, or nadir), a life expectancy of $>3$ months, satisfactory bone marrow function [white blood cell count $>2,0 \times 10^{9} / 1$; absolute neutrophil count $>1,5 \times 10^{9} / 1$; platelet count $>100 \times 10^{9} / 1$; hemoglobin $>10 \mathrm{~g} / \mathrm{dl}$ (if necessary after transfusion)], satisfactory renal function (serum creatinine $<1.25 \mathrm{x}$ upper norm of calculated creatinine clearance $>60 \mathrm{ml} / \mathrm{min}$ ) and satisfactory hepatic function (bilirubin $<1.25 \mathrm{x}$ upper norm or $<5 \mathrm{x}$ upper norm with hepatic metastasis; transaminases $<3 \times$ upper norm or $<5 \mathrm{x}$ upper norm with hepatic metastasis), and an Eastern Cooperative Oncology Group performance status $\leq 2$ as well as a left ventricular ejection fraction $\geq 50 \%$.

Patients were excluded from the trial, if one of the following criteria was met: Previous anthracycline treatment; secondary malignancy, with the exception of ductal carcinoma in situ or cervical intraepithelial neoplasia; patients lacking an evaluable or measurable tumor mass and not showing a significant increase of CA125; cardiac arrhythmias, cardiac insufficiency or history of myocardial infarction within the previous 6 months; acute infection not allowing cytotoxic treatment; severe comorbidities, such as uncontrolled infections; synchronous or scheduled radiotherapy; or major psychiatric diseases not allowing treatment within trial conditions.

Outcome assessment. The primary trial objective was observing the activity of non-pegylated liposomal doxorubicin in terms of response. The secondary objective was to evaluate toxicity, duration of response, overall survival (OS) time and progression-free survival (PFS) time.

Response was evaluated on every 3 therapy cycles by CT/MRI and CA125 response, as well as every 3 months during 18 months of follow-up. Evaluation of response was assessed according to the World Health Organization criteria (20), as follows: Complete remission, total disappearance of measurable tumor mass; partial remission, tumor mass reduction $>50 \%$; stable disease, reduction of tumor mass $<50 \%$ or progression $<25 \%$; or progressive disease, new tumor manifestations or progression of the tumor mass $>25 \%$ or increase of CA125 $>25 \%$ ).

The clinical benefit rate (CBR) was defined as the rate of patients with complete remission or partial remission, or with stable disease for $>6$ months. PFS was defined as the time from diagnosis to disease progression or recurrence, or to the date of mortality or last known contact (whichever occurred first.) OS was determined as time interval between randomization and mortality or last contact. Toxicity and tolerability analyses were performed in all patients who completed $\geq 1$ cycle of therapy. Hematological and non-hematological toxicities were evaluated and graded according to the National Cancer Institute (NCI) Common Toxicity Criteria (21). 
Data management and statistical analysis. All data were collected and saved using the electronic documentation system SAS version 9.2 (SAS Institute, Inc., Cary, NC, USA) according to good clinical practice in a pseudonymized form (22). Data are expressed as the absolute and relative frequencies, or the median and range. Data from survival analyses are presented as the mean or median survival rates and $95 \%$ confidence intervals. Fisher's exact test was used to compare statistical differences between patient subgroups. Statistical significance was considered to be indicated by $\mathrm{P}<0.05$. Data from toxicity analyses, PFS, OS and CBR were evaluated with methods of descriptive statistics. All analyses, except the primary endpoint, have an explorative character.

\section{Results}

Patients and treatments. Between February 2005 and January 2011, 29 patients with recurrent ovarian cancer within 12 months after platinum-containing primary treatment were randomized to this trial in nine German sites. The characteristics of the patients are presented in Table I. The median age of the patients was 60 years (range, 25-75 years). The majority of the patients had primarily been diagnosed with low-grade EOC (55.2\%) of International Federation of Gynecology and Obstetrics (FIGO) stage IIIC (48.3\%) (23). All but 3 patients initially presented with FIGO stage III/IV disease.

Second-line therapy with non-pegylated liposomal doxorubicin consisted of $75 \mathrm{mg} / \mathrm{m}^{2}$ given every 3 weeks as 1-h i.v. infusions for a total of 6-8 cycles. As grade III hematological toxicities occurred in 3 of the first 5 treated patients, the treatment schedule was modified to $60 \mathrm{mg} / \mathrm{m}^{2} \mathrm{~d} 1 \mathrm{q} 22$. Within the subsequent 24 patients, only 1 patient required a dose reduction to $50 \mathrm{mg} / \mathrm{m}^{2}$ according to protocol after the third cycle of chemotherapy, due to a non-hematological toxicity.

A total of 124 cycles of non-pegylated liposomal doxorubicin were administered. There were 11 patients who completed $\geq 6$ cycles of therapy, and 4 patients who completed 8 cycles of chemotherapy, respectively. Early discontinuation of therapy was necessary in 15 cases (due to progressive disease in $46.7 \%$ of the cases and due to non-hematological toxicities in $20 \%$, respectively).

Activity data. Of the included patients, 20 were available for response evaluation (10 with initial relapse at $<6$ months after platinum-containing therapy, and 10 patients with initial relapse 6-12 months after platinum-containing therapy). There were 9 patients who were not available for response evaluation, mostly due to premature discontinuation of therapy because of progression, toxicity or personal decision following the first cycle of therapy. The overall response rate of the patients, all of whom initially had measurable disease, was 20\%; 1 patient (initial relapse within 6-12 months after platinum-containing therapy) experienced a complete remission, whereas 3 patients (15\%) experienced partial remission (all 3 patients with initial relapse within 6-12 months after platinum-containing therapy). A further 6 patients $(30 \%)$ exhibited stable disease (3 patients with initial relapse $<6$ months after platinum-containing therapy; 3 patients with initial relapse within 6-12 months after
Table I. Patient characteristics $(n=29)$.

\begin{tabular}{lc}
\hline Parameter & Value \\
\hline Age, years; median (range) & $60(25-75)$ \\
Histology of primary tumor, $\mathrm{n}$ & \\
Adenocarcinoma & 3 \\
Serous carcinoma & 8 \\
Papillary serous carcinoma & 12 \\
Clear cell carcinoma & 1 \\
Other & 5
\end{tabular}

\section{Grade, $n$}

1

2

3

$\mathrm{X}$

Primary tumor FIGO stage, $\mathrm{n}$ I 2

IIA

IIB

1

IIIA

2

IIIB

IIIC 14

IV 5

Missing data 1

WHO performance status prior to start of Myocet ${ }^{\circledR}$ chemotherapy, $n$ 0

1

Missing data

Duration of first-line pretreatment $112(72-234)$ with platinum, days; median (range)

FIGO, International Federation of Gynecology and Obstetrics; WHO, World Health Organization.

platinum-containing therapy), consistent with a clinical benefit rate of $50 \%$. In 8 patients $(40 \%)$, a progression of the disease was observed (5 patients with initial relapse $<6$ months after platinum-containing therapy; 3 patients with initial relapse within 6-12 months after platinum-containing therapy). Table II shows all response data in detail. Serum levels of CA125 during the study period were available for 25 patients; in summary, the median serum levels of CA125 were $246 \mathrm{U} / \mathrm{ml}$ [standard deviation $(\mathrm{SD}), \pm 2,260.76 \mathrm{U} / \mathrm{ml}$ ] prior to treatment, $231.60 \mathrm{U} / \mathrm{ml}(\mathrm{SD}, \pm 530.38 \mathrm{U} / \mathrm{ml})$ at the end of treatment, and $116.3 \mathrm{U} / \mathrm{ml}(\mathrm{SD}, \pm 617.3)$ at the end of the 18-month follow-ups.

A total of 25 patients were available for progress evaluation in follow-up: 22 of these patients (88\%) showed progression of disease (11 patients with initial relapse $<6$ months after platinum-containing therapy; 11 patients with initial relapse within 6-12 months after platinum-containing therapy). The median PFS was 3.45 months (SD, \pm 0.74 ) (3.45 months in patients with initial relapse $<6$ months after 
Table II. Clinical response rates and survival data (entire study population, $\mathrm{n}=29)$.

\begin{tabular}{lccr}
\hline & \multicolumn{2}{c}{ Relapse $(\mathrm{n}=20), \mathrm{n}$} & \\
\cline { 2 - 3 } Clinical response & $<6$ months & $6-12$ months & $\%$ \\
\hline Complete remission & 0 & 1 & 5 \\
Partial remission & 0 & 3 & 15 \\
Stable disease & 3 & 3 & 30 \\
Progressive disease & 5 & 3 & 40 \\
Not evaluable & 2 & 0 & 10 \\
\hline
\end{tabular}

Table III. Toxicities in the patient population $(\mathrm{n}=29)$.

\begin{tabular}{lrrr}
\hline & \multicolumn{2}{c}{ Grade, $\mathrm{n}$} & \\
\cline { 2 - 3 } Parameter & I/II & III/IV & Total \\
\hline Hematological & & & \\
Neutropenia & 16 & 1 & 17 \\
Leukopenia & 17 & 1 & 18 \\
Anemia & 28 & 1 & 29 \\
Thrombocytopenia & 14 & 0 & 14 \\
Non-hematological & & & \\
Allergic reaction & 2 & 3 & 4 \\
Nausea & 14 & 1 & 15 \\
Vomiting & 4 & 0 & 4 \\
Alopecia & 0 & 0 & 0 \\
Fever & 2 & 0 & 2 \\
Fatigue & 9 & 2 & 11 \\
Mucositis/stomatitis & 2 & 0 & 2 \\
\hline
\end{tabular}

platinum-containing therapy; 3.45 months in patients with initial relapse within 6-12 months after platinum-containing therapy). The median OS was 10.2 months (SD, \pm 1.39 ) (9.04 months in patients with initial relapse $<6$ months after platinum-containing therapy; 11.47 patients with initial relapse within 6-12 months after platinum-containing therapy). A total of 10 patients were still alive at last follow-up. Further chemotherapy was administered in $61.9 \%$ of the patients during follow-up; topotecan was the most common subsequently administered chemotherapy, with a median of 3 cycles. Additionally, $7.1 \%$ of patients underwent a second surgery during follow-up. Fig. 1 demonstrates the corresponding Kaplan-Meier analyses for PFS and OS.

Toxicity. All 29 patients were evaluable for toxicity. A total of 105 adverse events occurred. The majority of the adverse events were documented in the NCI groups of 'blood/bone marrow', 'gastrointestinal' and 'constitutional symptoms'. Grade III/IV toxicity was predominantly documented for hematological toxicities: Anemia, neutropenia and leukopenia grade III were documented for 1 patient each, whereas leukopenia and low granulocytes grade I/II were common, occurring in $17(58.6 \%)$ and $16(55.2 \%)$ patients, respectively. Anemia grade I/II was
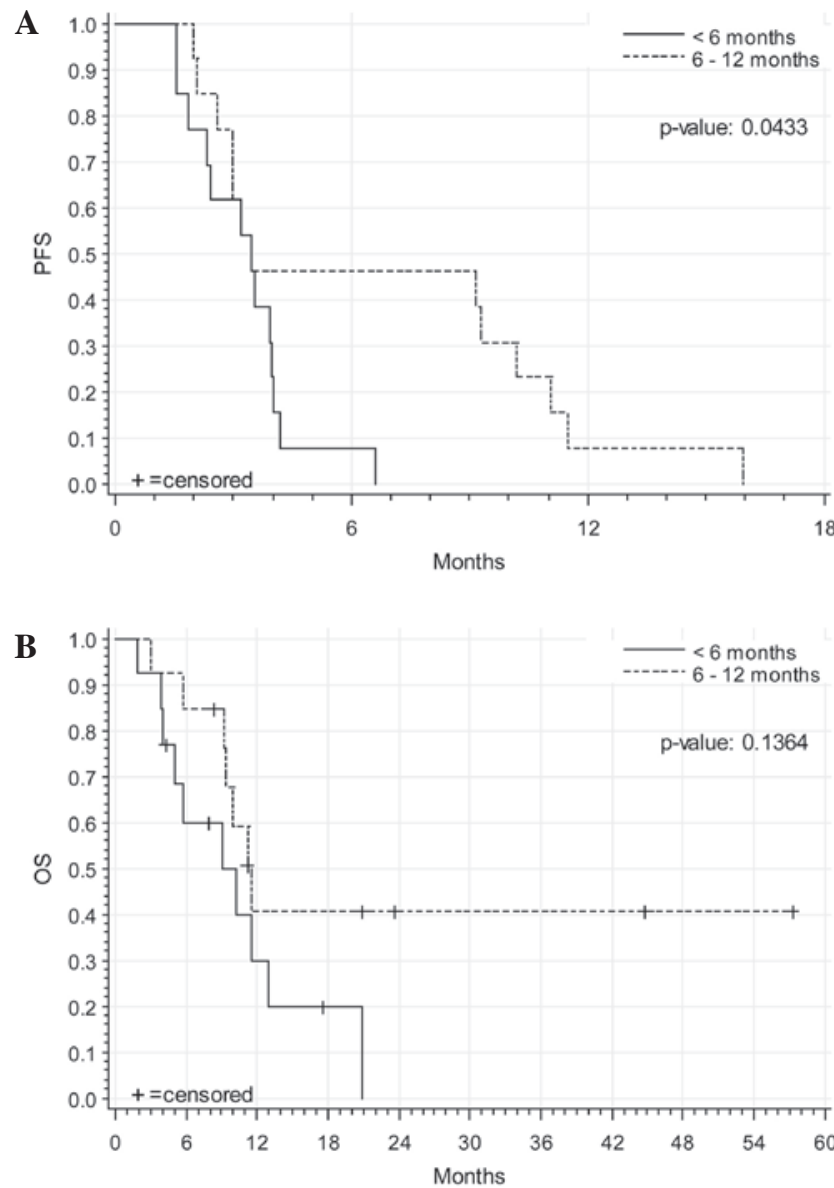

Figure 1. Kaplan-Meier analyses of (A) PFS and (B) OS of all studied and evaluable patients $(n=25)$. PFS, progression-free survival; OS, overall survival.

documented in 28 patients. Thrombocytopenia grade I/II was documented for 14 patients. Blood transfusions were given in 9 cases $(7.4 \%)$ and growth factors in 26 cases $(21.5 \%)$.

The most common non-hematological toxicity was nausea (grade I/II, 14 patients; grade III, 1 patient), and fatigue as a constitutional symptom was documented in 9 patients for grade I/II and in 2 patients for grade III. No PPE was observed in any patient. There were 3 patients with grade III allergic reactions (flush) on infusion that could easily be managed by steroid infusions. There was 1 grade III increase of aspartate aminotransferase/alanine aminotransferase. All of the other adverse events documented were of a lower grade. Table III summarizes the observed hematological and non-hematological toxicities observed.

A total of 12 severe adverse events occurred. In 9 cases, hospitalization was the reason for the evaluation as 'severe'. Mortality due to thrombosis during therapy occurred in 1 patient; however, this fatality was doubtfully related to the treatment. The median duration of the severe adverse events was 3 days.

\section{Discussion}

Despite significant improvements in primary treatment efforts for EOC, over two-thirds of these patients relapse and require a second-line therapy (3). In particular, for patients with non-platinum-sensitive disease, there is an urgent 
necessity for developing drugs that offer satisfactory antitumoral activity as well as mild toxicity, in order to maintain an acceptable quality of life.

The current trial demonstrated for the first time prospectively that non-pegylated liposomal doxorubicin is active in the treatment of recurrent ovarian cancer. With a response rate of $20 \%$, the activity of this drug is similar and comparable to other single-agent treatment options in this setting (7-9). In addition, PFS and OS times of 3.5 and 10.2 months, respectively, are also consistent with previously reported survival data from other second-line trials (11).

An advantage of non-pegylated liposomal doxorubicin is the specific, moderate toxicity profile of the drug. Following dose modification during the trial to a $60 \mathrm{mg} / \mathrm{m}^{2} \mathrm{~d} 1 \mathrm{q} 22$ schedule, few grade III/IV hematological toxicities were subsequently observed in the patients. Furthermore, no alopecia or PPE were noted, indicating a remarkable clinical difference against pegylated liposomal doxorubicin treatment $(16,17)$, in which PPE can be expected in $~ 50 \%$ of cases at standard dosages of $50 \mathrm{mg} / \mathrm{m}^{2} \mathrm{q} 4 \mathrm{w}$, and grade III PPE may develop in $20 \%$ of the patients (17).

A clinical particularity of non-pegylated liposomal doxorubicin that was indicated from the current trial data was that there appears to be a relevant allergenic potential of the drug (3 patients experienced a grade III allergic reaction). This confirms previously reported experiences, and should lead the treating physician to specific caution in this respect (24). Nevertheless, this adverse reaction occurred only in $10 \%$, and was clinically uncomplicatedly manageable, resolving completely without any residuals. A pathophysiological explanation of this phenomenon is currently lacking; it can only be speculated that the presence of pegylating proteins on the surface of the liposomes may cover their allergenic potential, as this adverse event is not commonly reported from pegylated liposomal doxorubicin.

In summary, non-pegylated liposomal doxorubicin appears to be a useful additional treatment option for patients with recurrent non-platinum-sensitive disease.

\section{Acknowledgements}

This trial was realized with a scientific grant of Cephalon, Inc. (Teva Pharmaceutical Industries, Ltd., Frazer, PA, USA)

\section{References}

1. Siegel R, Ma J, Zou Z and Jemal A: Cancer statistics, 2014. CA Cancer J Clin 64: 9-29, 2014

2. Hennessy BT, Colemann RL and Markmann M: Ovarian Cancer Lancet 374: 1371-1382, 2009.

3. Armstrong DK: Relapsed ovarian cancer: Challenges and management strategies for a chronic disease. Oncologist 7 (Suppl 5): 20-28, 2002.

4. Piccart MJ, Du Bois A, Gore ME, Neijt JP, Pecorelli S and Pujade-Lauraine E: A new standard of care for treatment of ovarian cancer. Eur J Cancer 36: 10-12, 2000.

5. Bookman MA: Standard treatment in advanced ovarian cancer in 2015: The state of the art. Int J Gynecol Cancer 15 (Suppl 3): S212-S220, 2005
6. du Bois A, Herrstedt J, Hardy-Bessard AC, Müller HH, Harter P, Kristensen G, Joly F, Huober J, Avall-Lundqvist E, Weber B, et al: Phase III trial of carboplatin plus paclitaxel with or without gemcitabine in first-line treatment of epithelial ovarian cancer. J Clin Oncol 28: 4162-4169, 2010.

7. Naumann RW and Coleman RL: Management strategies for recurrent platinum-resistant ovarian cancer. Drugs 71: 1397-1412, 2011.

8. Pfisterer J and Ledermann JA: Management of platinum-sensitive recurrent ovarian cancer. Semin Oncol 33 (2 Suppl 6): S12-S16, 2006.

9. Cormio G, Loizzi V, Gissi F, Camporeale A, De Mitri P, Leone L, Putignano G and Selvaggi L: Long-term topotecan therapy in recurrent or persistent ovarian cancer. Eur J Gynaecol Oncol 32: 153-155, 2011

10. Markman M: Pegylated liposomal doxorubicin: Appraisal of its current role in the management of epithelial ovarian cancer. Cancer Manag Res 3: 219-225, 2011.

11. Gordon AN, Granai CO, Rose PG, Hainsworth J, Lopez A, Weissman C, Rosales R and Sharpington T: Phase II study of liposomal doxorubicin in platinum-and paclitaxel-refractory epithelial ovarian cancer. J Clin Oncol 18: 3093-3100, 2000.

12. Muggia FM, Hainsworth JD, Jeffers S, Miller P, Groshen S, Tan M, Roman L, Uziely B, Muderspach L, Garcia A, et al: Phase II study of liposomal doxorubicin in refractory ovarian cancer: Antitumor activity ad toxicity modification by liposomal encapsulation. J Clin Oncol 15: 987-993, 1997.

13. Israel VP, Garcia AA, Roman L, Muderspach L, Burnett A, Jeffers S and Muggia FM: Phase II study of liposomal doxorubicin in advanced gynecological cancers. Gynecol Oncol 78: 143-147, 2000

14. Campos SM, Penson RT, Mays AR, Berkowitz RS, Fuller AF, Goodman A, Matulonis UA, Muzikansky A and Seiden MV: The clinical utility of liposomal doxorubicin in recurrent ovarian cancer. Gynecol Oncol 81: 206-212, 2001.

15. Markman M, Kennedy A, Webster K, Peterson G, Kulp B and Belinson J: Phase 2 trial of liposomal doxorubicin $(40 \mathrm{mg} / \mathrm{m}(2))$ in platinum/paclitaxel-refractory ovarian and fallopian tube cancers and primary carcinoma of the peritoneum. Gynecol Oncol 78: 369-372, 2000.

16. Bernardi D, Errante D, Stefani $M$ and Salvagno L: Non-pegylated liposomal doxorubicin in metastatic breast cancer patients: A valuable therapeutic option requiring caution. Breast 19: 549-550, 2010.

17. Lorusso D, Di Stefano A, Carone V, Fagotti A, Pisconti S and Scambia G: Pegylated liposomal doxorubicin-related palmar-plantar erythrodysesthesia ('hand-foot' syndrome). Ann Oncol 18: 1159-1164, 2007.

18. Miolo G, Baldo P, Bidoli E, Lombardi D, Scalone S, Sorio R and Veronesi A: Incidence of palmar-plantar erythrodysesthesia in pretreated and unpretreated patients receiving pegylated liposomal doxorubicin. Tumori 95: 687-690, 2009.

19. Meier W, Römisch M and Hepp H: The value of reoperation in the treatment of ovarian cancer. Geburtshilfe Frauenheilkd 53: 30-34, 1993

20. World Health Organization: WHO handbook for reporting results of cancer treatment. WHO offset publication. No. 48 . World Health Organization, Geneva, Switzerland, 1979.

21. Cancer Therapy Evaluation Program: Common toxicity criteria for adverse events. Version 3.0, National Cancer Institute, National Institutes of Health, Bethseda, MD, 2006.

22. Food and Drug Administration: International Conference on Harmonisation; Good Clinical Practice Consolidated Guideline, 1997. https://www.gpo.gov/fdsys/pkg/FR-199705-09/pdf/97-12138.pdf. Accessed May 25, 2016.

23. Heintz AP, Odicino F, Maisonneuve P, Beller U, Benedet JL, Creasman WT, Ngan HY, Sideri M and Pecorelli S: Carcinoma of the ovary. J Epidemiol Biostat 6: 107-138, 2001.

24. Bokemeyer C and Lipp HP: Allergic reaction. In: Compendium of Oncology Standards in Diagnosis and Therapy. Schmoll HJ, Höffken K and Possinger K (eds). 4th edition. Springer-Verlag GmbH, Heidelberg. pp1947-1952, 2006 (In German). 